\title{
MULTIPARAMETER SPECTRAL THEORY AND TAYLOR'S JOINT SPECTRUM IN HILBERT SPACE
}

\author{
by B. P. RYNNE \\ (Received 23rd June 1986)
}

\section{Introduction}

Let $n \geqq 1$ be an integer and suppose that for each $i=1, \ldots, n$, we have a Hilbert space $H_{i}$ and a set of bounded linear operators $T_{i}, V_{i j}: H_{i} \rightarrow H_{i}, j=1, \ldots, n$. We define the system of operators

$$
W_{i}(\lambda)=T_{i}-\sum_{j=1}^{n} \lambda_{j} V_{i j}: H_{i} \rightarrow H_{i}, \quad i=1, \ldots, n,
$$

where $\lambda=\left(\lambda_{1}, \ldots, \lambda_{n}\right) \in \mathbb{C}^{n}$. Coupled systems of the form (1.1) are called multiparameter systems and the spectral theory of such systems has been studied in many recent papers. Most of the literature on multiparameter theory deals with the case where the operators $T_{i}$ and $V_{i j}$ are self-adjoint (see [14]). The non self-adjoint case, which has received relatively little attention, is discussed in [12] and [13].

In this paper we discuss the definition of the spectrum of the multiparameter system (1.1) in the non self-adjoint situation. Two approaches to the definition of the multiparameter spectrum have been adopted previously. In one approach the spectrum is defined to be the set of points $\lambda \in \mathbb{C}^{n}$ for which each of the operators $W_{i}(\lambda), i=1, \ldots, n$, are singular. We will denote the spectrum obtained from this definition by $\sigma(W)$. This definition is simple and readily applicable to any multiparameter system of the form (1.1). The basic properties of the spectrum obtained from this definition are discussed in [12].

In an alternative approach the spectrum of the system (1.1) is defined via the joint spectrum of an associated set of commuting operators $\Gamma_{1}, \ldots, \Gamma_{n}$, defined on the tensor product space $H=\bigotimes_{i=1}^{n} H_{i}$. The spectrum obtained from this definition will be denoted by $\sigma(\Gamma)$. The operators $\Gamma_{i}$ exist when the system satisfies a certain solvability condition which will be discussed below. This method has been used very successfully for selfadjoint multiparameter systems satisfying various definiteness conditions. The basic theory of self-adjoint multiparameter systems is discussed in [14] using this method.

In the self-adjoint case, under appropriate conditions, the two definitions of the spectrum lead to the same set, i.e. $\sigma(W)=\sigma(\Gamma)$ (see Theorem 3.7 in [14]). Our goal in this paper is to find conditions which ensure that this result holds in the non self-adjoint situation. The importance of this result lies in the fact that a variety of complementary information about the spectrum can be derived using the two definitions. For instance, 
in the self-adjoint case, the first definition can be used to obtain geometric information about the structure and location of the spectrum (see [3]). Conversely the second definition, together with the theory of joint spectra, provides basic spectral information such as the compactness of the spectrum and a functional calculus. When the operators $\Gamma_{i}$ are self-adjoint a spectral theorem for the system (1.1) can be obtained from these operators (see [14]).

In this paper we will adopt the definition of the joint spectrum of a set of commuting operators given by Taylor in [16] and [17]. An alternative definition of the joint spectrum using commutative Banach algebras is discussed in [6]. However the Taylor spectrum has many features which make it superior to the Banach algebra definition (see [9]). The main advantage of the Taylor joint spectrum, for our purposes, is that, under certain conditions, we will be able to prove the equality $\sigma(W)=\sigma(\Gamma)$. Since the Banach algebra definition of the joint spectrum generally leads to a larger spectrum than the Taylor definition (see [16]) we could not, in general, expect to obtain this result using the Banach algebra spectrum. There are circumstances in which the Taylor spectrum and the Banach algebra spectrum coincide, for instance when the operators are normal (see [5]). Thus in the self-adjoint case this problem does not arise.

We note that McGhee has considered the relationship between the spectra $\sigma(W)$ and $\sigma(\Gamma)$, when $\sigma(\Gamma)$ is defined using the Banach algebra joint spectrum. It is shown in [13] that certain subsets of the two spectra coincide. However, these subsets need not form the complete spectrum. There is also a partial discussion of this question, using the Taylor spectrum, in [7].

In Section 2 we describe the Taylor definition of the joint spectrum of a set of commuting operators on a Banach space. Some other material that will be required later will also be discussed. In Section 3 we construct the commuting operators $\Gamma_{i}$ and state precisely the two definitions of the spectrum of the multiparameter system (1.1) that we will use. We then show that if a mapping $V$ associated with the array of operators $\left[V_{i j}\right]$ is invertible, then the two definitions lead to the same spectrum. In Section 4 we discuss the structure of the mapping $V$ and examine some conditions which ensure the invertibility of $V$. These conditions are expressed in terms of a determinantal operator on the tensor product space $H$, as is standard in multiparameter theory. Our main result is that if the system satisfies a generalization of the usual right definiteness condition then the two types of multiparameter spectrum coincide.

\section{The joint spectrum of several commuting operators}

In this section we briefly review Taylor's definition of the joint spectrum for $n$-tuples of bounded commuting operators as discussed in [17]. In addition we discuss some related material which will be required below.

Let $X$ be a complex Banach space and suppose that $a=\left(a_{1}, \ldots, a_{n}\right)$ is an $n$-tuple of bounded commuting operators on $X$. If $\sigma=\left(s_{1}, \ldots, s_{n}\right)$ is an $n$-tuple of indeterminates let $\Lambda[\sigma]$ denote the exterior algebra over $\mathbb{C}$ generated by $s_{1}, \ldots, s_{n}$. The set of elements of $\Lambda[\sigma]$ with degree $p$ will be written as $\Lambda^{p}[\sigma]$. Now define the sets $\Lambda[\sigma, X]=X \otimes \Lambda[\sigma]$ and $\Lambda^{p}[\sigma, X]=X \otimes \Lambda^{p}[\sigma]$, where $\otimes$ denotes the tensor product. The set $\Lambda[\sigma, X]=$ $\left\{\Lambda^{p}[\sigma, X]: p \geqq 0\right\}$ is a graded module (see [11]). An element $x \otimes s_{j_{1}} \wedge \cdots \wedge s_{j_{p}} \in \Lambda^{p}[\sigma, X]$ will be written as $x s_{j_{1}} \wedge \cdots \wedge s_{j_{p}}$, where $\wedge$ denotes the exterior product. 
For each $p$ satisfying $1 \leqq p \leqq n$ we let $J(p)$ denote the set of multi-indices of the form $\mathbf{j}=\left(j_{1}, \ldots, j_{p}\right)$, where the $j_{i}$ are integers satisfying $1 \leqq j_{1}<\cdots<j_{p} \leqq n$. It can be seen that there are $\left(\begin{array}{l}n \\ p\end{array}\right)$ multi-indices in the set $J(p)$, where $\left(\begin{array}{l}n \\ p\end{array}\right)$ denotes the binomial coefficient $n ! / p !(n-p) !$. For any $\mathbf{j} \in J(p)$ we let $\hat{\mathbf{j}}$ denote the unique complementary multi-index in $J(n-p)$ such that $\mathrm{j} \cup \hat{\mathrm{j}}=\{1,2, \ldots, n\}$. If $1 \leqq p \leqq n$ then any element $\psi$ of $\Lambda^{p}[\sigma, X]$ can be written in the form

$$
\psi=\sum_{\mathbf{j}} x_{\mathrm{j}} s_{j_{1}} \wedge \cdots \wedge s_{j_{p}}
$$

where the summation is over all the elements $\mathbf{j} \in J(p)$. Using this representation it can be seen that $\Lambda^{p}[\sigma, X]$ can be canonically identified with the direct sum of $\left(\begin{array}{l}n \\ p\end{array}\right)$ copies of the space $X$. Also $\Lambda^{0}[\sigma, X]$ can be identified with $X$, while if $p>n$ then $\Lambda^{p}[\sigma, X]=0$. In the following we will use these identifications, where convenient, without further comment. In particular, if $L$ is a bounded linear operator on $X$ then $L$ can be defined in the obvious manner on $\Lambda[\sigma, X]$.

Now define the bounded linear maps $\delta^{p}(a): \Lambda^{p}[\sigma, X] \rightarrow \Lambda^{p+1}[\sigma, X], p=0,1, \ldots$, by

$$
\delta^{p}(a)\left(x s_{j_{1}} \wedge \cdots \wedge s_{j_{p}}\right)=\sum_{k=1}^{n}\left(a_{k} x\right) s_{k} \wedge s_{j_{1}} \wedge \cdots \wedge s_{j_{p}}
$$

and extend this to $\Lambda^{p}[\sigma, X]$ by linearity. It can easily be shown, using the commutativity of the operators $a_{1}, \ldots, a_{n}$, that

$$
\delta^{p+1}(a) \circ \delta^{p}(a)=0, \quad p \geqq 0 .
$$

Thus the sequence

$$
0 \longrightarrow \Lambda^{0}[\sigma, X] \stackrel{\delta^{0}(a)}{\longrightarrow} \Lambda^{1}[\sigma, X] \stackrel{\delta^{1}(a)}{\longrightarrow} \cdots \stackrel{\delta^{n-1}(a)}{\longrightarrow} \Lambda^{n}[\sigma, X] \longrightarrow 0
$$

is a cochain complex with coboundary operators $\delta^{p}(a), p \geqq 0$ (see [10]). This complex will be denoted by $F(X, a)$. The cohomology of the complex $F(X, a)$ is the graded module $H(X, a)=\left\{H^{p}(X, a): p \geqq 0\right\}$, where $H^{p}(X, a)$ is the quotient space $\operatorname{ker} \delta^{p}(a) / \operatorname{im} \delta^{p-1}(a)$ for $p \geqq 1$ and $H^{0}(X, a)=\operatorname{ker} \delta^{0}(a)$. Note that equation (2.1) implies that im $\delta^{p-1}(a) \subset \operatorname{ker} \delta^{p}(a)$ for all $p \geqq 0$.

The complex $F(X, a)$ is said to be exact if $\operatorname{ker} \delta^{0}(a)=0$ and im $\delta^{p}(a)=\operatorname{ker} \delta^{p+1}(a)$, for all $p \geqq 0$. It follows from this definition that the complex $F(X, a)$ is exact if and only if $H^{p}(X, a)=0$ for all $p \geqq 0$.

Definition. The $n$-tuple of operators $a$ on $X$ is said to be non-singular if the complex $F(X, a)$ is exact, otherwise it is said to be singular. If $\lambda \in \mathbb{C}^{n}$ then $\lambda$ is in the spectrum of $a$ on $X$ if and only if the tuple $a-\lambda=\left(a_{1}-\lambda_{1}, \ldots, a_{n}-\lambda_{n}\right)$ is singular. The spectrum of $a$ on $X$ will be denoted by $\operatorname{Sp}(a, X)$.

Various subsets of the spectrum of $a$ on $X$ can also be defined. For instance a point $\lambda \in \mathbb{C}^{n}$ is said to be in the joint point spectrum of $a$ if there exists a non zero $x \in X$ such that $\left(a_{i}-\lambda_{i}\right) x=0 i=1, \ldots, n$. The vector $x$ will be called a common eigenvector of the 
tuple $a$. Using the canonical representation of $\Lambda[\sigma, X]$ it can be seen that

$$
\delta^{0}(a-\lambda) x=\left(\left(a_{1}-\lambda_{1}\right) x, \ldots,\left(a_{n}-\lambda_{n}\right) x\right)=(0, \ldots, 0), \quad x \neq 0,
$$

and so $a-\lambda$ is singular. Thus the joint point spectrum is a subset of $\operatorname{Sp}(a, X)$. Other subsets of the spectrum can be defined in a similar manner, however only the joint point spectrum will be required below.

We now consider certain transformations of $\Lambda[\sigma, X]$ and $F(X, a)$. Let $X$ and $Y$ be Banach spaces and $\sigma=\left(s_{1}, \ldots, s_{n}\right), \tau=\left(t_{1}, \ldots, t_{m}\right)$ be tuples of indeterminates. Suppose that $u^{0}: X \rightarrow Y$ is a bounded operator and $\left[u_{i j}\right]$ is an $m \times n$ array of bounded linear operators on $Y$ with the property

$$
u_{i j} u_{k l}=u_{k l} u_{i j}, 1 \leqq i, k \leqq m, 1 \leqq j, l \leqq n, i \neq k,
$$

i.e. operators from different rows of the array $\left[u_{i j}\right]$ commute. A transformation $u: \Lambda[\sigma, X] \rightarrow \Lambda[\tau, Y]$ can be defined by

$$
u\left(\sum_{j} x_{j} s_{j_{1}} \wedge \cdots \wedge s_{j_{p}}\right)=\sum_{\mathbf{j}} u^{0}\left(x_{j}\right) u\left(s_{j_{1}}\right) \wedge \cdots \wedge u\left(s_{j_{p}}\right),
$$

where

$$
u\left(s_{j}\right)=u_{1 j} t_{1}+\cdots+u_{m j} t_{m}, \quad j=1, \ldots, n,
$$

and

$$
y u_{i j}=u_{i j}(y) \quad \text { for all } y \in Y .
$$

The transformation $u$ is a graded module homomorphism of degree zero i.e. for each $p \geqq 0, u$ maps $\Lambda^{p}[\sigma, X]$ into $\Lambda^{p}[\tau, Y]$. We let $u^{p}$ denote the restriction of $u$ to $\Lambda^{p}[\sigma, X]$. A transformation defined in this way is said to be a "special transformation" determined by the operators $\left(u^{0},\left[u_{i j}\right]\right)$. Special transformations of the above type are discussed in [17] where it is assumed that all the operators in the array $\left[u_{i j}\right]$ commute, rather than merely operators from different rows commuting. However the structure of the transformation is such that two operators from the same row of the array never act on the same element, thus the above commutativity assumption is sufficient to define the transformation unambiguously.

Note that if $\mathbf{j} \in J(p), x \in X$, and $u$ is as above then

$$
\begin{aligned}
u\left(x s_{j_{1}} \wedge \cdots \wedge s_{j_{p}}\right) & =u^{0}(x) u\left(s_{j_{1}}\right) \wedge \cdots \wedge u\left(s_{j_{p}}\right) \\
& =\sum_{i_{1} \cdots i_{p}}\left(u_{i_{1} j_{1}} \cdots u_{i_{p} j_{p}} u^{0}(x)\right) t_{i_{1}} \wedge \cdots \wedge t_{i_{p}} \\
& \sum_{i \in J(p)}\left(\operatorname{det} u_{i_{j}}\right) u^{0}(x) t_{i_{1}} \wedge \cdots \wedge t_{i_{p}},
\end{aligned}
$$

where det $u_{\mathrm{ij}}$ denotes the operator on $Y$ obtained by formally expanding the determinant of the $p \times p$ array of operators $\left[u_{i_{k} j}\right]_{l} k_{, l} l=1$ in the usual manner. The above commutativity 
assumption on the array $\left[u_{i j}\right]$ ensures that this definition is unambiguous. We note that the final equality in (2.2) is a consequence of the properties of the exterior product $\wedge$. The relationship between determinants and exterior algebra is discussed, in the scalar case, in section XVI.7 of [11]. Our commutativity assumptions on the operator arrays are sufficient to ensure that the discussion in [11] is valid in the present situation.

Now suppose that $b=\left(b_{1}, \ldots, b_{m}\right)$ is a tuple of bounded commuting operators on $Y$ and $u: \Lambda[\sigma, X] \rightarrow \Lambda[\tau, Y]$ is a graded module homomorphism of degree zero. We say that $u$ is a cochain map from $F(X, a)$ to $F(Y, b)$ if the diagram

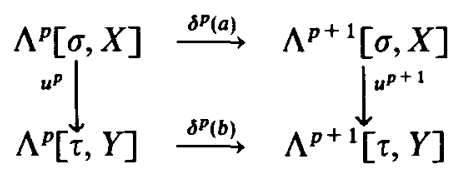

is commutative for each $p \geqq 0$. If this is so $u$ induces a homomorphism $u^{*}: H(X, a) \rightarrow H(Y, b)$ of cohomology (see [10]).

The following lemma is proved in [17] for commutative arrays $\left[u_{i j}\right]$. It can easily be seen that the proof given in [17] is valid with our commutativity assumptions.

Lemma 2.1. Let $u: \Lambda[\sigma, X] \rightarrow \Lambda[\tau, Y]$ be a special transformation and let $a=$ $\left(a_{1}, \ldots, a_{n}\right), b=\left(b_{1}, \ldots, b_{m}\right)$ be commuting tuples of operators on $X$ and $Y$ respectively. Then $u$ is a cochain map from $F(X, a)$ to $F(Y, b)$ if and only if the diagram

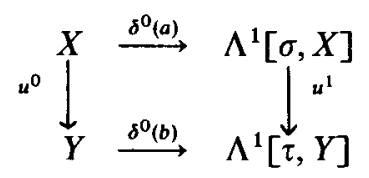

is commutative.

\section{Multiparameter spectral theory}

We now return to the multiparameter spectral problem outlined in Section 1. In order to proceed further we will transform this problem to a tensor product setting. Let $H=\bigotimes_{i=1}^{n} H_{i}$ denote the Hilbert space tensor product of the spaces $H_{i}$ (see [14]). Given a bounded linear operator $A_{i}: H_{i} \rightarrow H_{i}$ we can define an operator $A_{i}^{+}: H \rightarrow H$ as follows: let

$$
A_{i}^{+}\left(x_{1} \otimes \cdots \otimes x_{i} \otimes \cdots \otimes x_{n}\right)=x_{1} \otimes \cdots \otimes A_{i} x_{i} \otimes \cdots \otimes x_{n}
$$

for decomposable elements of $H$, and extend this definition to all of $H$ by linearity and continuity. The operator $A_{i}^{+}$is a bounded linear operator on $H$ (see [14]). We note that if $A_{i}: H_{i} \rightarrow H_{i}, A_{j}: H_{j} \rightarrow H_{j}, i \neq j$, are operators on different spaces then the operators $A_{i}^{+}$, $A_{j}^{+}$on $H$ commute.

Using this construction we can consider the system (1.1) in the space $H$, i.e. we have

$$
W_{i}^{+}(\lambda)=T_{i}^{+}-\sum_{j=1}^{n} \lambda_{j} V_{i j}^{+}: H \rightarrow H, \quad i=1, \ldots, n .
$$


We now define the determinantal operator

$$
\Delta=\operatorname{det}\left[V_{i j}^{+}\right]_{i, j=1}^{n}: H \rightarrow H,
$$

using the formal expansion of the determinant. Again this definition is unambiguous since the operators from different rows of the array $\left[V_{i j}^{+}\right]$commute.

From now on we will assume that the operator $\Delta$ has a bounded inverse on $H$. Assumptions on the invertibility of determinantal operators are commonplace in multiparameter theory. In the self adjoint case stronger definiteness conditions are usually used in place of invertibility (see [14]).

Now consider the system of equations

$$
\sum_{j=1}^{n} V_{i j}^{+} u_{j}=f_{i}, \quad i=1, \ldots, n
$$

where $u_{j}, f_{i} \in H$. We say that this system is solvable if there exists a unique solution $\left(u_{1}, \ldots, u_{n}\right)$ for each set of vectors $\left(f_{1}, \ldots, f_{n}\right)$. If it is known that a solution exists we can use Cramer's rule to solve (3.3) giving

$$
u_{j}=\Delta^{-1} \sum_{i=1}^{n} \hat{\Delta}_{i j} f_{i}, \quad j=1, \ldots, n,
$$

where $\hat{\Delta}_{i j}$ denotes the cofactor of $V_{i j}^{+}$in the determinantal expansion of $\Delta$ (see [8]). Thus the invertibility of $\Delta$ implies that if a solution of (3.3) exists it must be unique. Sufficient conditions for the solvability of equation (3.3) will be discussed below, for now we will assume that it is solvable.

If we define the operators

$$
\Gamma_{j}=\Delta^{-1} \sum_{i=1}^{n} \widehat{\Delta}_{i j} T_{i}^{+}: H \rightarrow H, \quad j=1, \ldots, n,
$$

and put $f_{i}=T_{i}^{+} u, i=1, \ldots, n$, in (3.3) for any $u \in H$, then it follows from the formula (3.4) for the solution of (3.3) that

$$
T_{i}^{+}=\sum_{j=1}^{n} V_{i j}^{+} \Gamma_{j}, \quad i=1, \ldots, n
$$

In addition it can be shown that the operators $\Gamma_{i}, i=1, \ldots, n$, commute (see [14]), ([14] discusses self-adjoint operators however the proof of the commutativity of the $\Gamma_{i}$ 's does not involve the self adjointness). We let $\Gamma$ denote the tuple of commuting operators $\left(\Gamma_{1}, \ldots, \Gamma_{n}\right)$.

We now consider the definition of a spectrum for the multiparameter system (1.1). To motivate the definition we first discuss the eigenvalues of the system.

Definition. A point $\lambda \in \mathbb{C}^{n}$ is said to be an eigenvalue of the system (1.1) if for each $i$ 
there exists a vector $x_{i} \in H_{i}, x_{i} \neq 0$, such that

$$
W_{i}(\lambda) x_{i}=0, \quad i=1, \ldots, n .
$$

It is obvious that if (3.7) holds then we have

$$
W_{i}^{+}(\lambda) x=0, \quad i=1, \ldots, n,
$$

where $x=x_{1} \otimes \cdots \otimes x_{n} \in H$. Conversely if (3.8) holds for some $x \in H$ it can be shown that there exists a set of vectors $x_{i} \in H_{i}$ for which (3.7) holds (see [14]). Thus defining the eigenvalues of (1.1) using (3.7) in the original space or using (3.8) in the tensor product space leads to the same set of points.

Now suppose that (3.8) holds for some $\lambda \in \mathbb{C}^{n}$ and $x \in H$ and rewrite it as

$$
T_{i}^{+} x=\sum_{j=1}^{n} V_{i j}^{+}\left(\lambda_{j} x\right), \quad i=1, \ldots, n .
$$

It follows from the unique solvability of the system (3.3) that (3.9) holds if and only if

$$
\Gamma_{i} x=\lambda_{i} x, \quad i=1, \ldots, n .
$$

Thus $\lambda$ is an eigenvalue of (1.1) if and only if $\lambda$ belongs to the joint point spectrum of the tuple of operators $\Gamma$. The vector $x$ is the corresponding common eigenvector.

The above discussion shows that the eigenvalues of the multiparameter system can be defined in terms of the original operators $W_{i}$ or in terms of the joint point spectrum of the tuple $\Gamma$. Either definition produces the same set of points in $\mathbb{C}^{n}$. We now attempt to define a multiparameter spectrum which has the same property.

Definition. The spectrum $\sigma(W)$ of the system (1.1) is the set of points $\lambda \in \mathbb{C}^{n}$ such that none of the operators $W_{i}(\lambda), i=1, \ldots, n$, has a bounded inverse.

Definition. The spectrum $\sigma(\Gamma)$ of the system (1.1) is the joint spectrum $\operatorname{Sp}(\Gamma, H)$ of the commuting tuple $\Gamma=\left(\Gamma_{1}, \ldots, \Gamma_{n}\right)$ on $H$.

The definition of the set $\sigma(W)$ is a simple generalization of the above definition of the eigenvalues of the system. This definition of the spectrum is the one used in [12]. Clearly the set $\sigma(W)$ contains the set of eigenvalues of the system. In addition the above discussion, together with the results of Section 2, shows that the set $\sigma(\Gamma)$ also contains the set of eigenvalues of the system.

We will now attempt to find conditions which will guarantee that $\sigma(W)=\sigma(\Gamma)$. By considering translations of the spectral parameter $\lambda$ it can easily be shown, using the relations in Theorem 6.2 .2 of [2], that it is sufficient to consider the point $\lambda=0$, i.e. we wish to show that $0 \in \sigma(W) \Leftrightarrow 0 \in \sigma(\Gamma)$.

Lemma 3.1. $0 \in \sigma(W)$ if and only if the commuting tuple $T=\left(T_{1}^{+}, \ldots, T_{n}^{+}\right)$is singular. 
Proof. By definition $0 \in \sigma(W)$ if and only if each of the operators $T_{i}$ is not boundedly invertible. It is shown in [4] that the joint spectrum of the tuple $T$ is given by the Cartesian product

$$
\mathrm{Sp}(T, H)=\ddot{X}_{i=1}^{n} \mathrm{Sp}\left(T_{i}, H_{i}\right)
$$

where $\operatorname{Sp}\left(T_{i}, H_{i}\right)$ equals the usual spectrum of $T_{i}$ as an operator in $H_{i}$. Hence $0 \in \operatorname{Sp}(T, H)$ if and only if each operator $T_{i}$ is not invertible. This proves the lemma.

Lemma 3.1. shows that to prove the equality of $\sigma(W)$ and $\sigma(\Gamma)$ it is sufficient to show that the tuple $T$ is non-singular if and only if the tuple $\Gamma$ is non-singular. In order to do this we construct a special transformation $V: \Lambda[\sigma, H] \rightarrow \Lambda[\sigma, H]$, as defined in Section 2, by choosing $u^{0}=V^{0}=I$, the identity operator on $H$, and $u_{i j}=V_{i j}^{+}$. Since the operators from different rows of the array $\left[V_{i j}^{+}\right]$are derived from operators on different spaces they commute, thus the array satisfies the conditions required to define a special transformation.

Lemma 3.2. The transformation $V: \Lambda[\sigma, H] \rightarrow \Lambda[\sigma, H]$ is a cochain map from $F(H, \Gamma)$ to $F(H, T)$.

Proof. It follows from Lemma 2.1 that it is sufficient to prove the commutativity of the diagram

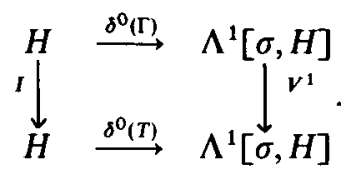

We noted in Section 2 that $\Lambda^{1}[\sigma, H]$ can be identified with $H^{n}$ and, with this identification, we have

$$
\delta^{0}(T)=\left(T_{1} x, \ldots, T_{n} x\right), \delta^{0}(\Gamma) x=\left(\Gamma_{1} x, \ldots, \Gamma_{n} x\right), \quad \text { for each } x \in H .
$$

Also $V^{1}$ is equivalent to the transformation

$$
\left(x_{1}, \ldots, x_{n}\right) \rightarrow\left(\sum_{j=1}^{n} V_{1 j}^{+} x_{j}, \ldots, \sum_{j=1}^{n} V_{n j}^{+} x_{j}\right)
$$

on $H^{n}$. Thus equation (3.6) shows that

$$
V^{1} \delta^{0}(\Gamma) x=\delta^{0}(T) x
$$

for all $x \in H$, and so the above diagram is commutative.

By considering formula (2.2) describing the structure of a special transformation it can 
be seen that the mapping $V^{n}$ on $\Lambda^{n}[\sigma, H] \simeq H$ is equivalent to the mapping $\Delta$ on $H$. Also it follows from the representation (3.11) of the mapping $V^{1}$ that the invertibility of $V^{1}$ is equivalent to the solvability of equation (3.3). Thus, if $V$ is invertible, then $\Delta$ is invertible and the system (3.3) is solvable, which implies that the operators $\Gamma_{i}$ exist and the set $\sigma(\Gamma)$ is defined.

We are now ready to prove our main theorem.

Theorem 3.3. If the array $\left[V_{i j}\right]$ is such that the special transformation $V: \Lambda[\sigma, H] \rightarrow \Lambda[\sigma, H]$ is invertible then the spectra $\sigma(W)$ and $\sigma(\Gamma)$ of the multiparameter system (1.1) are identical.

Proof. The preceding discussion has reduced the proof of the theorem to proving the following proposition: the complex $F(H, T)$ is exact if and only if the complex $F(H, \Gamma)$ is exact. To prove this we consider the cochain mapping $V: F(H, \Gamma) \rightarrow F(H, T)$ defined above. Since $V$ is a cochain mapping it induces a homomorphism $V^{*}: H(H, \Gamma) \rightarrow H(H, T)$ of cohomology. The invertibility of the mapping $V$ shows that the homomorphism $V^{*}$ is an isomorphism, i.e. the restrictions $V^{* p}: H^{p}(H, \Gamma) \rightarrow H^{p}(H, T)$ are isomorphisms between the cohomology groups for each $p \geqq 0$. Now, by definition, a complex is exact if and only if all the cohomology groups of the complex are zero. Since the cohomology groups of the complexes $F(H, T)$ and $F(H, \Gamma)$ are isomorphic it follows that exactness of either complex implies the exactness of the other.

We observe that the proof of Theorem 3.3 proves rather more than the equality of the sets $\sigma(W)$ and $\sigma(\Gamma)$. The structure of the cohomology module associated with a singular tuple of operators is closely related to the type of singularity of the tuple (see p. 176 of [16]). For instance the elements of the 0th cohomology module correspond to common eigenvectors of the tuple of operators. The proof of Theorem 3.3 shows that when $V$ is invertible the cohomology modules $H(H, T)$ and $H(H, \Gamma)$ are isomorphic. Thus, if $\lambda$ belongs to the spectrum of the system (1.1), then the commuting tuples $\left(W_{1}^{+}(\lambda), \ldots, W_{n}^{+}(\lambda)\right)$ and $\left(\Gamma_{1}-\lambda_{1}, \ldots, \Gamma_{n}-\lambda_{n}\right)$ have the same type of singularity in the sense that their cohomology modules are isomorphic.

We now consider the case $n=1$. In thise case we have $W(\lambda)=T-\lambda V, \lambda \in \mathbb{C}$ (where we have omitted the subscripts in this case), and, by definition, $\Delta=V$. Also, if $V$ is invertible, then $\Gamma=V^{-1} T$. In this case it is obvious that $W(\lambda)$ is not invertible if and only if $\lambda$ is in the spectrum of $\Gamma$. Thus, in this sense, the invertibility condition in Theorem 3.3 is a generalization of the condition that the operator $V$ be invertible in the 1-parameter case.

We can now illustrate the usefulness of the equality $\sigma(W)=\sigma(\Gamma)$ and the use of the theory of the joint spectrum to provide information about the multiparameter spectrum. It is shown in [16] that the joint spectrum of a set of commuting operators is nonempty and compact. Thus, when $V$ is invertible, Theorem 3.3 shows that $\sigma(W)$ is nonempty and compact. Now, it is shown in [12], under fairly general hypotheses, that the set $\sigma(W)$ is closed. However, without further conditions on the system, the set $\sigma(W)$ can be empty or unbounded. In [13] it is shown that if $\Delta$ is invertible and the system (3.3) is solvable than a certain subset of $\sigma(W)$ is equal to a non-empty subset of the set $\sigma(\Gamma)$. 
Thus in this case the set $\sigma(W)$ is non-empty. However, without the equality $\sigma(W)=\sigma(\Gamma)$ it is not easy to deduce that the set $\sigma(W)$ is compact.

\section{Invertibility conditions}

In this section we consider the structure of the mapping $V$ in more detail and discuss some conditions which ensure that $V$ is invertible. We begin by deriving a more convenient representation of the mapping.

As noted in Section 2 the set $\Lambda^{p}[\sigma, H]$ consists of the direct sum of $\left(\begin{array}{l}n \\ p\end{array}\right)$ copies of the space $H$, one for each multi-index $\mathbf{j} \in J(p)$. By the construction of the transformation $V$ it follows that

$$
V\left(\sum_{i} x_{j} s_{j_{1}} \wedge \cdots \wedge s_{j_{p}}\right)=\sum_{i} \sum_{j}\left(\operatorname{det} V_{i j}\right) x_{j} s_{i_{1}} \wedge \cdots \wedge s_{i_{p}},
$$

see equation (2.2). Thus the invertibility of $V$ is equivalent to the unique solvability of the system

$$
\sum_{\mathbf{j}}\left(\operatorname{det} V_{\mathrm{ij}}\right) x_{\mathbf{j}}=y_{\mathrm{i}}, \quad \mathrm{i} \in J(p)
$$

for all sets of vectors $\left\{y_{\mathbf{i}} \in H: \mathbf{i} \in J(p)\right\}$, for each $p=1, \ldots, n$.

In order to establish the invertibility of $V$ we will reformulate this problem using the ideas of Section 2. For each $j=1, \ldots, n$, we define the column tuple $C_{j}=\left(V_{1 j}^{+}, \ldots, V_{n j}^{+}\right)$. From the construction of the operators $V_{i j}^{+}$on the tensor product space $H$ it follows that the operators in the tuples $C_{j}$ are commutative, and so the coboundary operators $\delta^{p}\left(C_{j}\right): \Lambda^{p}[\sigma, H] \rightarrow \Lambda^{p+1}[\sigma, H]$ can be defined as in Section 2. These operators have the following commutativity property:

$$
\delta^{p+1}\left(C_{j}\right) \circ \delta^{p}\left(C_{k}\right)=\delta^{p+1}\left(C_{k}\right) \circ \delta^{p}\left(C_{j}\right), \quad 1 \leqq j, k \leqq n .
$$

for all $p \geqq 0$. This result can easily be verified using the fact that the operators from different rows of the array $\left[V_{i j}^{+}\right]$commute. In addition we have

$$
\Delta x=\delta^{n-1}\left(C_{n}\right) \circ \cdots \circ \delta^{0}\left(C_{1}\right) x, \quad x \in H .
$$

It can now be seen, using the relationship between determinants and exterior algebra, that for each $p=1, \ldots, n$, the system (4.1) can be rewritten in the equivalent form

$$
\sum_{j} \delta^{p-1}\left(C_{j_{p}}\right) \cdots \circ \delta^{0}\left(C_{j_{1}}\right) x_{j}=\phi
$$

where $x_{\mathrm{i}} \in H$ and $\phi \in \Lambda^{p}[\sigma, H]$. The mapping $V$ is invertible if, for each $p=1, \ldots, n$, the system (4.4) is uniquely solvable for all $\phi \in \Lambda^{p}[\sigma, H]$.

We begin the discussion of equation (4.4) by considering the uniqueness of the solution. Also, if a commutativity condition is satisfied, then the invertibility of $V$ can be 
established easily. We will say that the array $\left[V_{i j}\right]$ is commutative if the operators in each row of the array commute with each other. Obviously this implies that all the operators in the array $\left[V_{i j}^{+}\right]$commute.

Lemma 4.1. If the operator $\Delta$ is invertible then the mapping $V$ is injective. If, in addition, the array $\left[V_{i j}\right]$ is commutative then $V$ is invertible.

Proof. Suppose that, for some $p=1, \ldots, n$, equation (4.4) has a solution for some $\phi \in \Lambda^{p}[\sigma, H]$. Then, for any $\mathbf{j} \in J(p)$, we can operate on (4.4) with the operator $\delta^{n-1}\left(C_{\hat{j}_{n-p}}\right) \circ \cdots \circ \delta^{p}\left(C_{j_{1}}\right)$ and, using equations (2.1), (4.2) and (4.3), it can be seen that we obtain the equations

$$
\Delta x_{\mathrm{j}}=\delta^{n-1}\left(C_{\hat{j}_{n-p}}\right) \circ \cdots \circ \delta^{p}\left(C_{\hat{j}_{1}}\right) \phi, \quad \mathbf{j} \in J(p) .
$$

Thus the invertibility of $\Delta$ implies that

$$
x_{\mathrm{j}}=\Delta^{-1} \delta^{n-1}\left(C_{\tilde{j}_{n-p}}\right) \circ \cdots \circ \delta^{p}\left(C_{\tilde{j}_{1}}\right) \phi, \quad \mathbf{j} \in J(p) .
$$

This shows that, for each $p=1, \ldots, n$, and $\phi \in \Lambda^{p}[\sigma, H]$, the solution of (4.4) (if it exists) is unique and is given by (4.6). Thus $V$ is injective. Now suppose that the array $\left[V_{i j}\right]$ is commutative. It can be seen that the commutativity of the operators in the array $\left[V_{i j}^{+}\right]$ ensures that the operator $\Delta^{-1}$ commutes with the coboundary operators $\delta^{p}\left(C_{j}\right)$. Substituting (4.6) into (4.4) and using (4.2) and (4.3), together with the commutativity, shows that (4.6) is in fact a solution of (4.4) in this case. Hence $V$ is invertible.

We remark that the proof of Theorem 3.3 can be modified to show that if $V$ is injective and the complex $F(H, T)$ is exact then the complex $F(H, \Gamma)$ is exact. This shows that when $V$ is injective we have $\sigma(\Gamma) \subset \sigma(W)$. Lemma 4.1 now implies the following corollary.

Corollary 4.2. If the operator $\Delta$ is invertible then $\sigma(\Gamma) \subset \sigma(W)$.

We will now derive an equivalent expression for the solution of the system (4.4) that does not involve the coboundary operators explicitly. Define the operators

$$
\operatorname{cof} V_{\mathrm{ij}}=S_{\mathrm{ij}} \operatorname{det} V_{\hat{\mathrm{i}}}, \quad \mathbf{i}, \mathbf{j} \in J(p), \quad p=1, \ldots, n,
$$

where $S_{\mathrm{ij}}$ is the sign factor $(-1)^{\operatorname{sgn} i}(-1)^{\operatorname{sgn} \mathbf{j}}$ and $\operatorname{sgn} \mathbf{i}$ denotes the parity of the transformation

$$
(1, \ldots, p, p+1, \ldots, n) \rightarrow\left(i_{1}, \ldots, i_{p}, \hat{i}_{1}, \ldots, \hat{i}_{n-p}\right) .
$$

Using the Laplace expansion of a determinant (see equation (34) on p. 550 of [11]) it can be shown that

$$
\sum_{\mathbf{k}}\left(\operatorname{cof} V_{\mathbf{k} j}\right)\left(\operatorname{det} V_{\mathbf{k} j}\right)= \begin{cases}\Delta, & \text { if } \mathbf{i}=\mathbf{j} \\ 0, & \text { if } \mathbf{i} \neq \mathbf{j}\end{cases}
$$


(we note that the formal properties of operator determinants of the form we are considering are discussed in Chapter 6 of [2]). Using these relations we can obtain the following result in a similar manner to the proof of Lemma 4.1.

Lemma 4.3. If the operator $\Delta$ is invertible then, for each $p=1, \ldots, n$, the solution of (4.1) (if it exists) is given by

$$
x_{\mathrm{j}}=\Delta^{-1} \sum_{\mathrm{i}}\left(\operatorname{cof} V_{\mathrm{ij}}\right) y_{\mathrm{i}}, \quad \mathrm{j} \in J(p) .
$$

This result is a generalization of the Cramer's rule formula (3.4) for the solution of the system (3.3). The two formulae coincide when $p=1$.

Combining Theorem 3.3 and Lemma 4.1 proves the following result.

Corollary 4.4. Suppose that the array $\left[V_{i j}\right]$ is commutative and the determinant $\Delta$ is invertible. Then the multiparameter spectra $\sigma(W)$ and $\sigma(\Gamma)$ are identical.

In addition, when the array $\left[V_{i j}\right]$ is commutative, the question of the invertibility of $\Delta$ on the tensor product space $H$ can be reduced to considering the joint spectra of the rows of the array $\left[V_{i j}\right]$ in the original spaces. We define the commuting tuples $R_{i}=\left(V_{i 1}, \ldots, V_{i n}\right), i=1, \ldots, n$.

Theorem 4.5. If the array $\left[V_{i j}\right]$ is commutative then the determinantal operator $\Delta$ is invertible if and only if

$$
0 \notin\left\{a \in \mathbb{R}: a=\operatorname{det}\left[a_{i j}\right], \text { where }\left(a_{i 1}, \ldots, a_{i n}\right) \in \mathrm{Sp}\left(R_{i}, H_{i}\right), \quad i=1, \ldots, n\right\} .
$$

Proof. It is shown in [4] that the joint spectrum of the commuting set of operators $\left[V_{i j}^{+}\right]$on $H$ is given by the cartesian product

$$
\operatorname{Sp}\left(\left[V_{i j}^{+}\right], H\right)=\stackrel{X}{i=1}_{x} \operatorname{Sp}\left(R_{i}, H_{i}\right) \subset \mathbb{C}^{n^{2}} .
$$

Thus the result follows from the spectral mapping theorem for the analytic functional calculus for sets of commuting operators (see [17]).

We now consider the non-commutative situation. In the case where each of the spaces $H_{i}$ is finite dimensional Atkinson uses the Cramer's rule formula (3.4) together with a dimensional argument to show that (3.3) is solvable when $\Delta$ is invertible (see [2]). A similar argument, using the formula (4.8), also shows that $V$ is invertible when $\Delta$ is invertible in the finite dimensional case. Thus in this case we again have the equality $\sigma(W)=\sigma(\Gamma)$ when $\Delta$ is invertible. Note also that in the finite dimensional case the Taylor joint spectrum coincides with the joint point spectrum (see [5]).

In the infinite dimensional case the problem is harder. In fact in [1] an example is constructed for which $\Delta$ is invertible but the system (3.3) is not solvable. Thus the invertibility of $\Delta$ is not a sufficient condition for the invertibility of $V$ in general. 
However if we let $\overline{W(\Delta)}$ denote the closure of the numerical range of the operator $\Delta$ the following theorem is effectively Theorem 1 of [1].

Theorem 4.6. Suppose that $0 \notin \overline{W(\Delta)}$. Then the system (3.3) is solvable.

The condition that $0 \notin \overline{W(\Delta)}$ is a generalization of the usual positivity condition used to establish solvability of (3.3) in the case of an array of self-adjoint operators (see [8]). We will now show that this condition also implies that $V$ is invertible. Note that the condition ensures that $\Delta$ is invertible and hence, by Lemma 4.1, it is sufficient to show that there exists a solution of (4.4) for each $p=1, \ldots, n$, and all $\phi \in \Lambda^{p}[\sigma, H]$. We will prove the result by a sequence of lemmas.

Lemma 4.7 If $\Delta$ is invertible and the condition

$$
\operatorname{im} \delta^{p-1}\left(C_{p}\right) \circ \cdots \circ \delta^{0}\left(C_{1}\right)=\left\{\psi \in \Lambda^{p}[\sigma, H]: \delta^{p}\left(C_{j}\right) \psi=0, \quad j=1, \ldots, p\right\}
$$

is satisfied for each $p=1, \ldots, n$, then $V$ is invertible.

Proof. For each $p=1, \ldots, n$, let $\mathbf{p}$ denote the multi-index $(1,2, \ldots, p)$. We can rewrite (4.4) in the form

$$
\delta^{p-1}\left(C_{p}\right) \circ \cdots \circ \delta^{0}\left(C_{1}\right) x_{\mathrm{p}}=\phi-\sum_{\mathrm{j} \neq \mathrm{p}} \delta^{p-1}\left(C_{j_{p}}\right) \circ \cdots \circ \delta^{0}\left(C_{j_{1}}\right) x_{\mathrm{j}} .
$$

It follows from the hypothesis in the lemma that a sufficient condition for this equation to have a solution $x_{\mathrm{p}} \in H$ is that the following system of equations in $\Lambda^{p+1}[\sigma, H]$ be satisfied:

$$
\sum_{\mathbf{j} \neq \mathbf{p}} \delta^{p}\left(C_{r}\right) \circ \delta^{p-1}\left(C_{j_{p}}\right) \circ \cdots \circ \delta^{0}\left(C_{j_{1}}\right) x_{\mathrm{j}}=\delta^{p}\left(C_{r}\right) \phi, \quad r=1, \ldots, p .
$$

Equation (2.1) implies that, for each $r=1, \ldots, p$, the summation in (4.10) need only be over those multi-indices $\mathrm{j} \in J(p)$ for which $r \neq j_{k}, k=1, \ldots, p$. For each $r$ the order of the operators on the left hand side of (4.10) can be rearranged, using (4.2), to bring the equation to the form (4.4) with summation over a restricted subset of the set of multi-indices $J(p+1)$. The above procedure can now be repeated, for each equation in the system (4.10), to obtain a new system of equations in the space $\Lambda^{p+2}[\sigma, H]$ such that when this new system is satisfied each equation in the system (4.10) has a solution. Continuing this procedure we eventually find that a sufficient condition for the existence of the solution $x_{p}$ of (4.9) is the solvability of a set of equations of the form

$$
\delta^{n-1}\left(C_{n}\right) \circ \cdots \circ \delta^{0}\left(C_{1}\right) x_{j}=\delta^{n-1}\left(C_{\hat{j}_{n-p}}\right) \circ \cdots \circ \delta^{p}\left(C_{j_{p}}\right) \phi
$$

where $\mathbf{j}$ ranges over a subset of the set $J(p)$. However the solvability of these equations, for all $\phi \in \Lambda^{p}[\sigma, H]$, follows from the invertibility of $\Delta$ (using equation (4.3)). Thus we have shown that (4.2) has a solution for all $\phi \in \Lambda^{p}[\sigma, H]$. This shows that $V$ is invertible. 
Lemma 4.8. Suppose that $\Delta$ is invertible and the condition

$$
\left\{\psi \in \Lambda^{p}[\sigma, H]: \delta^{p}\left(C_{j}\right) \psi=0, j=1, \ldots, n\right\}=\{0\}
$$

is satisfied for each $p=0, \ldots, n-1$. Then, for each $p=1, \ldots, n$, and all $\mathbf{j} \in J(p)$, we have

$$
\operatorname{im} \delta^{p-1}\left(C_{j_{p}}\right) \circ \cdots \circ \delta^{0}\left(C_{j_{1}}\right)=\left\{\psi \in \Lambda^{p}[\sigma, H]: \delta^{p}\left(C_{j_{r}}\right) \psi=0, r=1, \ldots, p\right\} .
$$

Proof. The invertibility of $\Delta$ implies that im $\delta^{n-1}\left(C_{n}\right) \circ \cdots \circ \delta^{0}\left(C_{1}\right)=\Lambda^{n}[\sigma, H]$, by (4.3), and aiso $\delta^{n}\left(C_{j}\right) \psi=0$ for all $\psi \in \Lambda^{n}[\sigma, H], j=1, \ldots, n$, by the definition of the coboundary operators. This proves the lemma in the case $p=n$.

The inclusions

$$
\operatorname{im} \delta^{p-1}\left(C_{j_{p}}\right) \circ \cdots \circ \delta^{o}\left(C_{j_{1}}\right) \subset\left\{\psi \in \Lambda^{p}[\sigma, H]: \delta^{p}\left(C_{j_{r}}\right) \psi=0, r=1, \ldots, p\right\}, \quad p=1, \ldots, n,
$$

are obvious from equations (2.1) and (4.2), so we must prove the reverse inclusions.

Equations (4.2) and (4.3) and the invertibility of $\Delta$ shows that, for each $p=1, \ldots, n-1$, and any $\mathbf{j} \in J(p)$, the operator $\Delta^{-1} \delta^{n-1}\left(C_{\hat{j}_{n-p}}\right) \circ \cdots \circ \delta^{p}\left(C_{\hat{j}_{1}}\right)$ is a left inverse of the operator $\delta^{p-1}\left(C_{j_{p}}\right) \circ \cdots \circ \delta^{o}\left(C_{j_{1}}\right)$. Thus we have the direct sum decompositions

$$
\begin{aligned}
& \Lambda^{p}[\sigma, H]=\operatorname{ker} \delta^{n-i}\left(C_{j_{n-p}}\right) \circ \cdots \circ \delta^{p}\left(C_{\hat{j}_{1}}\right) \oplus \operatorname{im} \delta^{p-1}\left(C_{j_{p}}\right) \circ \cdots \circ \delta^{0}\left(C_{j_{1}}\right), \\
& p=1, \ldots, n-1, \quad \mathbf{j} \in J(p)
\end{aligned}
$$

(this follows from Theorem 12.9 on p. 251 of [15]).

We now consider the case $p=n-1$. Suppose that there exists $\xi \in \Lambda^{n-1}[\sigma, H]$ such that $\xi \neq 0, \delta^{n-1}\left(C_{j}\right) \xi=0, \quad j=1, \ldots, n-1$, and $\xi \notin \operatorname{im} \delta^{n-2}\left(C_{n-1}\right) \circ \cdots \circ \delta^{0}\left(C_{1}\right)$. Then it follows from the decomposition (4.12) that $\xi$ can be represented uniquely in the form $\xi=\psi+\theta$, where $\quad \psi \in \operatorname{ker} \delta^{n-1}\left(C_{n}\right)$ and $\theta \in \operatorname{im} \delta^{n-2}\left(C_{n-1}\right) \circ \cdots \circ \delta^{0}\left(C_{1}\right)$. Since $\xi \notin \operatorname{im} \delta^{n-2}\left(C_{n-1}\right) \circ \cdots \circ \delta^{0}\left(C_{1}\right)$ we must have $\psi \neq 0$. Also, since $\theta \in \operatorname{im} \delta^{n-2}\left(C_{n-1}\right) \circ \cdots \circ \delta^{0}\left(C_{1}\right)$, we have $\delta^{n-1}\left(C_{j}\right) \theta=0, j=1, \ldots, n-1$, by $(2.1)$, so it follows from the choice of $\xi$ that $\delta^{n-1}\left(C_{j}\right) \psi=0, j=1, \ldots, n-1$. The hypothesis of the lemma now implies that $\psi=0$, which is a contradiction. This proves the lemma in the case $p=n-1$ and $\mathbf{j}=(1,2, \ldots, n-1)$. The result can be proved similarly for all $\mathbf{j} \in J(n-1)$.

Now let $p=n-2$ and $\mathbf{j}=(1,2, \ldots, n-2)$. Using a similar argument to the above, we suppose that there exists a non-zero $\psi \in \operatorname{ker} \delta^{n-1}\left(C_{n}\right) \circ \delta^{n-2}\left(C_{n-1}\right)$ such that $\delta^{n-2}\left(C_{j}\right) \psi=0$, $j=1, \ldots, n-2$. If we let $\phi=\delta^{n-2}\left(C_{n-1}\right) \psi \in \Lambda^{n-1}[\sigma, H]$, then $\phi \in \operatorname{ker} \delta^{n-1}\left(C_{n}\right)$ since $\psi \in \operatorname{ker} \delta^{n-1}\left(C_{n}\right) \circ \delta^{n-2}\left(C_{n-1}\right)$. Also $\delta^{n-1}\left(C_{n-1}\right) \phi=0$ by $(2.1)$, and $\delta^{n-1}\left(C_{j}\right) \phi=$ $\delta^{n-1}\left(C_{n-1}\right) \circ \delta^{n-2}\left(C_{j}\right) \psi=0, j=1, \ldots, n-2$, by $(4.2)$ and the choice of $\psi$. Hence it follows from the argument in the case $p=n-1$ that $\phi=0$. Similarly we can show that $\delta^{n-2}\left(C_{n}\right) \psi=0$. This shows that $\delta^{n-2}\left(C_{j}\right) \psi=0, j=1, \ldots, n$, and so, by the hypothesis, $\psi=0$. This proves the lemma when $p=n-2$ and $j=(1,2, \ldots, n-2)$. Again the result for all $\mathbf{j} \in J(n-2)$ can be proved similarly. This argument can be continued in a similar manner for $p=n-3, n-4, \ldots, 1$, to prove the general result. 
Lemma 4.9. Suppose that $0 \notin \overline{W(\Delta)}$. Then

$$
\left\{\psi \in \Lambda^{p}[\sigma, H]: \delta^{p}\left(C_{j}\right) \psi=0, j=1, \ldots, n\right\}=\{0\},
$$

for each $p=0, \ldots, n-1$.

Proof. We begin by transforming the array of operators $\left[V_{i j}\right]$ into a new array of the form

$$
\left[\begin{array}{cccc}
V_{11}-\frac{\alpha_{1}}{\alpha_{n}} V_{1 n} & \ldots & V_{1, n-1}-\frac{\alpha_{n-1}}{\alpha_{n}} V_{1 n} & V_{1 n} \\
\vdots & & \vdots & \vdots \\
V_{n 1}-\frac{\alpha_{1}}{\alpha_{n}} V_{n n} & \ldots & V_{n, n-1}-\frac{\alpha_{n-1}}{\alpha_{n}} V_{n n} & V_{n n}
\end{array}\right]
$$

where the $\alpha_{i}, i=1, \ldots, n$, are complex numbers with $\alpha_{n} \neq 0$. This new array has the same determinant $\Delta$ as the original array $\left[V_{i j}\right]$. Now let $\Delta_{n-1}$ and $\Delta_{n-1}^{t}$ denote the determinants of the sub-arrays formed from the first $n-1$ rows and columns of the original and transformed arrays respectively. Using the method of proof of Theorem 2 of [8] it can be shown that the numbers $\alpha_{i}$ can be chosen in such a way that $0 \notin \overline{W\left(\Delta_{n-1}^{t}\right)}$. Note that we have assumed that this construction produces a non-zero $\alpha_{n}$. However this does not entail any loss of generality since at least one of the $\alpha_{i}$ 's must be non-zero (see [8]) and we may renumber the columns to ensure that $\alpha_{n} \neq 0$. The columns of the transformed array are of the form $C_{j}^{t}=C_{j}-\left(\alpha_{j} / \alpha_{n}\right) C_{n}, j \neq n$, and it can be seen that

$$
\begin{aligned}
\delta^{p+1}\left(C_{j}^{t}\right) \circ \delta^{p}\left(C_{k}^{t}\right)= & \delta^{p+1}\left(C_{j}\right) \circ \delta^{p}\left(C_{k}\right)-\left(\alpha_{j} / \alpha_{n}\right) \delta^{p+1}\left(C_{j}\right) \circ \delta^{p}\left(C_{n}\right) \\
& -\left(\alpha_{k} / \alpha_{n}\right) \delta^{p+1}\left(C_{n}\right) \circ \delta^{p}\left(C_{k}\right) .
\end{aligned}
$$

This result, together with (4.2), shows that the existence of a solution of the system of the form (4.4), derived from the transformed array, implies the existence of a solution of the original system. In view of this we will, from now on, assume that the array $\left[V_{i j}\right]$ is such that $0 \notin \overline{W(\Delta)}$ and $0 \notin \overline{W\left(\Delta_{n-1}\right)}$.

We will now prove the result by induction on $n$. It is clear that the result holds when $n=1$. Thus we assume that $n>1$ and the result holds for any $(n-1) \times(n-1)$ array of operators on a Hilbert space $H$ with the property that operators from different rows commute and whose determinant satisfies the hypothesis of the lemma. Note that in the proof we will only use these properties of the array, the tensor product structure is not needed. In particular this means that in the induction process we only use the space $H$, we do not need to consider the tensor product spaces of the form $\bigotimes_{i=1}^{r} H_{i}, r=1, \ldots, n$.

We let $\sigma^{\prime}$ denote the tuple of indeterminates $\left(s_{1}, \ldots, s_{n-1}\right)$ and construct the set $\Lambda\left[\sigma^{\prime}, H\right]$ as in Section 2 . For each $p=1, \ldots, n-1$, the set $\Lambda^{p}\left[\sigma^{\prime}, H\right]$ can be canonically identified with the subset of the set $\Lambda^{p}[\sigma, H]$ consisting of elements of the form

$$
\sum_{j \in J^{\prime}(p)} x_{j} s_{j_{1}} \wedge \cdots \wedge s_{j_{p}}
$$


where $J^{\prime}(p) \subset J(p)$ is the set of multi-indices $\mathrm{j}$ for which $j_{p} \neq n$. Also, if

$$
\psi=\sum_{j \in J^{\prime}(p)} x_{j} s_{j_{1}} \wedge \cdots \wedge s_{j_{p}} \in \Lambda^{p}\left[\sigma^{\prime}, H\right]
$$

then we let $\psi \wedge s_{n}$ denote the element

$$
\sum_{\mathbf{j} \in J^{\prime}(p)} x_{j^{\prime}} s_{j_{1}} \wedge \cdots \wedge s_{j_{p}} \wedge s_{n} \in \Lambda^{p+1}[\sigma, H] .
$$

Using this notation and the above identification it can be seen that, for any $p=0, \ldots, n$, any element $\psi \in \Lambda^{p}[\sigma, H]$ can be uniquely represented in the form

$$
\psi=\phi+\theta \wedge s_{n},
$$

where $\phi \in \Lambda^{p}\left[\sigma^{\prime}, H\right], \theta \in \Lambda^{p-1}\left[\sigma^{\prime}, H\right]$ (when $p=0$ we put $\phi=\psi$ and $\theta \wedge s_{n}=0$ ).

Now, for any $p=0, \ldots, n-2$, let $\psi \in \Lambda^{p}[\sigma, H]$ satisfy the equations

$$
\delta^{p}\left(C_{j}\right) \psi=0, \quad j=1, \ldots, n .
$$

If we define the commuting tuples $C_{j}^{\prime}=\left(V_{1 j}^{+}, \ldots, V_{n-1, j}^{+}\right), j=1, \ldots, n-1$, it follows from the representation (4.13) that

$$
\delta^{p}\left(C_{j}\right) \psi=\delta^{p}\left(C_{j}^{\prime}\right) \phi+(-1)^{p}\left(V_{n j}^{+} \phi\right) \wedge s_{n}+\left(\delta^{p-1}\left(C_{j}^{\prime}\right) \theta\right) \wedge s_{n}, \quad j=1, \ldots, n-1 .
$$

Hence equation (4.14) and the properties of the exterior algebra imply that

$$
\begin{gathered}
\delta^{p}\left(C_{j}^{\prime}\right) \phi=0, \quad j=1, \ldots, n-1, \\
(-1)^{p} V_{n j}^{+} \phi+\delta^{p-1}\left(C_{j}^{\prime}\right) \theta=0, \quad j=1, \ldots, n-1 .
\end{gathered}
$$

The induction hypothesis and equation (4.15) now imply that $\phi=0$. Thus equation (4.16) reduces to

$$
\delta^{p-1}\left(C_{j}^{\prime}\right) \theta=0, \quad j=1, \ldots, n-1,
$$

and hence, by our induction hypothesis, $\theta=0$. Thus $\psi=0$, which proves the result for $p=0, \ldots, n-2$.

To prove the result for $p=n-1$ we note that the canonical isomorphism between $\Lambda^{n-1}[\sigma, H]$ and $H^{n}$ allows us to rewrite equation (4.14) in the form

$$
\sum_{j=1}^{n} V_{j i}^{+} x_{j}=0, \quad i=1, \ldots, n \text {. }
$$

This system of equations is the transpose of the system (3.3). If we define the mapping $\Psi: H^{n} \rightarrow H^{n}$ by

$$
\Psi\left(x_{1}, \ldots, x_{n}\right)=\left(\sum_{j=1}^{n} V_{j 1}^{+} x_{j}, \ldots, \sum_{j=1}^{n} V_{j n}^{+} x_{j}\right),
$$


we see that equation (4.17) is equivalent to the equation $\Psi\left(x_{1}, \ldots, x_{n}\right)=0$. The adjoint of the mapping $\Psi$ is given by

$$
\Psi *\left(x_{1}, \ldots, x_{n}\right)=\left(\sum_{i=1}^{n} V_{1 i}^{+*} x_{i}, \ldots, \sum_{i=1}^{n} V_{n i}^{+*} x_{i}\right)
$$

Now, since the operator $\Delta^{*}$ is the determinant of the array $\left[V_{i j}^{+*}\right]$ and $0 \neq \overline{W(\Delta)}$ implies that $0 \notin W\left(\Delta^{*}\right)$, it follows from Theorem 4.6 that the mapping $\Psi^{*}$ is invertible. Hence the mapping $\Psi$ is invertible. Thus equation (4.17) implies that $\left(x_{1}, \ldots, x_{n}\right)=(0, \ldots, 0)$ and hence $\psi=0$. This proves the result for $p=n-1$ and completes the proof of the lemma.

Collecting together the results of Lemmas $4.7,4.8$ and 4.9 proves the following theorem.

Theorem 4.10. Suppose that $0 \notin \overline{W(\Delta)}$. Then the multiparameter spectra $\sigma(W)$ and $\sigma(\Gamma)$ are identical.

\section{REFERENCES}

1. M. S. Almamedov and G. A. Isaev, Solvability of nonselfadjoint linear operator systems and the set of decomposability of multiparameter spectral problems, Soviet. Math. Dokl. 31 (1986), 472-474.

2. F. V. Atkinson, Multiparameter Eigenvalue Problems Vol I: Matrices and Compact Operators (Academic Press, New York, 1972).

3. P. A. Binding and P. J. BRownE, A variational approach to multiparameter eigenvalue problems in Hilbert space, SIAM J. Math. Anal. 9 (1978), 1054-1067.

4. Z. Ceausescu and F. H. Vasilescu, Tensor products and the joint spectrum in Hilbert spaces, Proc. Amer. Math. Soc. 72 (1978), 505-508.

5. М. Сно and M. TaKaGuchi, Identity of Taylor's joint spectrum and Dash's joint spectrum, Studia Math. 70 (1982), 225-229.

6. A. T. DASH, Joint spectra, Studia Math. 45 (1973), 225-237.

7. A. КацLSTRom, Joint spectra and multiparameter eigenvalue problems, in Multiparameter Problems, ed. G. F. Roach (University of Strathclyde Seminars in Applied Mathematical Analysis, Shiva, 1984).

8. A. Kallstrom and B. D. Sleeman, Solvability of a linear operator system, J. Math. Anal. Appl. 55 (1976), 785-793.

9. A. YA. KhelemsKil, Homological methods in Taylor's holomorphic calculus of several operators in a Banach space, Russian Math. Surveys 36 (1981), 139-192.

10. S. MacLane, Homology (Springer-Verlag, Berlin, 1963).

11. S. MacLane and G. Birkoff, Algebra (MacMillan, New York, 1967).

12. D. F. MCGheE and G. F. RoACH, The spectrum of multiparameter problems in Hilbert space, Proc. Roy. Soc. Edinburgh 91A (1981), 31-42.

13. D. F. McGree, Multiparameter problems and joint spectra, Proc. Roy. Soc. Edinburgh 93A (1982), 129-135. 
14. B. D. Sleeman, Multiparameter Spectral Theory in Hilbert Space (Pitman, London, 1978).

15. A. E. TaYlor and D. C. LaY, Introduction to Functional Analysis (Wiley, New York, 1980).

16. J. L. TAYLOR, A joint spectrum for several commuting operators, J. Funct. Anal. 6 (1970), 172-191.

17. J. L. TAYLOR, The analytic functional calculus for several commuting operators, Acta. Math. 125 (1970), 1-38.

Department of Mathematics

UNIVERSITY OF YORK

HESLINGTON

YORK YO1 SDD 